REFERENCES:

[1] van der Helm-van Mil, A. H., et al. Arthritis and rheumatism, 2006. 54(4): p. $1117-1121$.

[2] van Steenbergen, H.W., et al. Ann Rheum Dis, 2017. 76(3): p. 491-496.

Disclosure of Interests: None declared

DOI: 10.1136/annrheumdis-2021-eular.2554

\section{POS0357 MIRNAs CORRELATE WITH IMPROVEMENT IN DISEASE ACTIVITY IN PATIENTS WITH RHEUMATOID ARTHRITIS ON TUMOUR NECROSIS FACTOR INHIBITORS}

T. David ${ }^{1,2}$, N. Nair ${ }^{3}$, J. Oliver ${ }^{3,4}$, E. Schordan ${ }^{5}$, H. Firat ${ }^{6}$, K. Hyrich $^{1,2,7}$ A. Morgan ${ }^{8,9}$, A. G. Wilson ${ }^{10}$, J. D. Isaacs ${ }^{11,12}$, D. Plant ${ }^{3}$, A. Barton ${ }^{1,2,3} .{ }^{1}$ Manchester Royal Infirmary, Manchester University NHS Foundation Trust, The Kellgren Centre for Rheumatology, Manchester, United Kingdom; ${ }^{2}$ The University of Manchester, NIHR Manchester Musculoskeletal Biomedical Research Unit, Manchester, United Kingdom: ${ }^{3}$ The University of Manchester, Centre for Musculoskeletal Research, Manchester, United Kingdom; ${ }^{4}$ Leeds Teaching Hospitals NHS Trust, Yorkshire and North East Genomic Laboratory Hub, Leeds, United Kingdom; ${ }^{5}$ FIRALIS S.A., CSO (Molecular Diagnostics), Huningue, France; ${ }^{6}$ FIRALIS S.A., CEO and CSO (Management Team), Huningue, France; ${ }^{7}$ The University of Manchester, Arthritis Research UK, Centre for Epidemiology, Centre for Musculoskeletal Research, Manchester Academic Health Sciences Centre, Manchester, United Kingdom; ${ }^{8}$ University of Leeds, Leeds Institute of Cardiovascular and Metabolic Medicine, Leeds, United Kingdom; ${ }^{9}$ Leeds Teaching Hospitals NHS Trust, NIHR Leeds Biomedical Research Centre, Leeds, United Kingdom; ${ }^{10}$ University College Dublin, UCD School of Medicine and Medical Science, Conway Institute, Dublin, Ireland; ${ }^{11}$ Newcastle University, Translational and Clinical Research Institute, Faculty of Medical Sciences, Newcastle upon Tyne, United Kingdom; ${ }^{12}$ Newcastle upon Tyne Hospitals NHS Foundation Trust, Musculoskeletal Unit, Newcastle upon Tyne, United Kingdom

Background: Tumour necrosis factor inhibitors (TNFi) although effective in the treatment of rheumatoid arthritis (RA), show a variable response rate. Therefore, there is a need to identify treatment response predictors to inform therapy selection in order to practise precision medicine. MicroRNAs (miRNAs) are endogenous, single-stranded, non-coding RNAs that can alter gene expression by regulating messenger RNA translation. There is evidence for miRNA involvement in RA pathogenesis and they may serve as a useful biomarker of treatment response. Objectives: To identify miRNAs associated with response to TNFi in RA. Methods: Biologic naïve patients were selected from the Biologics in Rheumatoid Arthritis Genetics and Genomics Study Syndicate (BRAGGSS), a prospective multi-center UK study investigating treatment response biomarkers to TNFi with a primary outcome measure of change in DAS28 scores. Patients were stratified into European League Against Rheumatism (EULAR) good or non-responders based on their 3 or 6-month DAS28-CRP score.

Pre-treatment and 3-month post-treatment serum samples were substrates for miRNA profiling, which was conducted by FIRALIS using the HTG EdgeSeq miRNA whole transcriptome $\mathrm{V} 2$ targeted sequencing assay. Linear modelling using $\mathrm{R}$ package limma compared miRNA expression at (i) pre-treatment and at three-months, in EULAR good-responders and non-responders (ii) longitudinal change in expression from pre-treatment to three-months in EULAR good and non-responders.

A literature search was conducted to identify miRNAs associated with RA as a diagnostic and/or treatment response predictor. Data on these miRNAs were extracted from the miRNAs identified in the serum samples. A correction for multiple testing was applied to statistical tests.

Results: A total of 54 patients were analysed; of these, 35 (65\%) were female, median disease duration [inter-quartile range] was 6 years [2 - 14] $(n=51)$, and $44 / 51(86 \%)$ patients were on a concomitant disease modifying anti-rheumatic drug. Of the 54 patients, $39(72 \%)$ were classified as EULAR good-responders and $15(28 \%)$ as non-responders. 1880 miRNAs were detected in the serum samples. 64 miRNAs were identified to be associated with RA from the literature, of which, 26 were identified in the serum samples tested.

No difference in pre-treatment or three-month miRNA levels was seen comparing EULAR good-responders and non-responders (FDR $\mathrm{p}<0.05)$. There was a significant differential expression of four miRNAs at 3-months in good-responders compared with pre-treatment levels; miR-125a-3p (downregulated, $p$-value 0.002), miR-149-3p (upregulated, $p$-value 0.004), miR-766-3p (downregulated, $p$-value 0.008), miR-146b-5p (upregulated, $p$-value 0.006). No significant differences were observed between 3-months and baseline in non-responders.

Conclusion: Although no pre-treatment miRNAs were associated with TNFi response, changes in the levels of four miRNAs were detected at 3-months compared to baseline in EULAR good-responders. Future work involves validation of these samples in a larger patient cohort and analysing miRNA levels at 6 and 12 months. Replication and validation of these results in larger studies are required to analyse the role of miRNAs in stratifying EULAR good-responders from non-responders at three-months, and as treatment response predictors to TNFi in RA

Acknowledgements: Joint last-author: Dr. Darren Plant

Disclosure of Interests: Trixy David: None declared, Nisha Nair: None declared, James Oliver: None declared, Eric Schordan: None declared, Hüseyin Firat None declared, Kimme Hyrich Consultant of: consultancy/honoraria from AbbVie, Grant/research support from: Pfizer, UCB, BMS, Ann Morgan: None declared, Anthony G Wilson: None declared, John D Isaacs Speakers bureau: consultancy/ speaker fees from AbbVie, Gilead, Roche, UCB, Consultant of: consultancy/ speaker fees from AbbVie, Gilead, Roche, UCB, Grant/research support from Pfizer, Darren Plant: None declared, Anne Barton: None declared DOI: 10.1136/annrheumdis-2021-eular.2841

\section{POS0358 EVALUATION OF THE EFFECT OF MTOR EXPRESSION ON THE CLINICAL MANIFESTATIONS OF KNEE OSTEOARTHRITIS IN OBESE AND NORMAL WEIGHT PATIENTS}

E. Strebkova ${ }^{1}$, E. Tchetina ${ }^{2}$, L. Alekseeva ${ }^{1} .{ }^{1}$ V. A. Nasonova Research Institute of Rheumatology, Osteoarthritis, Moscow, Russian Federation; ${ }^{2}$ V. A. Nasonova Research Institute of Rheumatology, Laboratory of Immunology and Molecular Biology of Rheumatic Diseases, Moscow, Russian Federation

Background: Currently, a large number of molecular biological and genetic markers are known to be involved in the development of osteoarthritis (OA) The mammalian target of rapamycin (mTOR) signaling pathway is responsible for chondrocyte proliferation, cartilage matrix production, and cell growth. OA is characterized by increased mTOR synthesis, which is accompanied by an increase in proliferative activity and destruction of chondrocytes. Obesity is an important factor in the progression of knee OA. The study of mTOR expression in patients with $\mathrm{OA}$ and obesity is an urgent task in the development of personalized OA therapy.

Objectives: To determine the expression of mTOR in patients with knee OA in combination with obesity and normal body weight. To evaluate the effect of mTOR on the clinical manifestations of OA in patients with different body mass index (BMI).

Methods: The study included 73 female patients aged $45-65$ y.o. with Kellgren-Lawrence stage II-III knee OA. The patients were divided into 2 groups: group $1(n=50)$ with obesity $(B M I>30 \mathrm{~kg} / \mathrm{cm} 2)$ and group $2(n=23)$ with normal or increased body weight (BMI $<30 \mathrm{~kg} / \mathrm{cm} 2)$. The average age of patients with obesity is $56.5 \pm 5.87$ years, without obesity $-58.7 \pm 5.43$ years. Clinical manifestations were evaluated by a WOMAC. RNA was isolated from the patients ' blood samples, which was used to determine the expression of mTOR.

Results: Patients with knee OA with and without obesity did not differ in age. OA develops at an earlier age in obese patients, than in non-obese patients $(p$ $<0.001$ ). Patients from 1 group had a high $\mathrm{BMl}>30 \mathrm{~kg} / \mathrm{m} 2$ at the onset of $\mathrm{OA}$ Obese patients had more severe knee OA is significantly more often detected: Kellgren-Lawrence stage III was determined in $10 \%$ of obese patients and in $4.35 \%$ - without obesity $(p<0.001)$. Significantly higher values of the WOMAC index pain, stiffness, joint functional failure, and total WOMAC were observed in obese patients $(p=0.006, p=0.039, p=0.037$, and $p=0.014$, respectively). Obese patients had higher VAS pain scores $(p<0.05)$ compared to patients with a lower BMI. Obese patients had a higher mTOR expression $(p<0.05)$ of $8.02 \pm 8.62$, compared to non-obese patients. High mTOR expression was associated with VAS knee pain $(r=0.78 ; p<0.05)$ and WOMAC pain $(r=0.89 ; p<0.05)$ in obese patients (Table 1).

Table 1. Correlation of m-TOR

\begin{tabular}{lcc}
\hline Parameters & mTOR $(1$ group, $n=50)$ & mTOR $(2$ group, $n=23)$ \\
\hline Body weight & $p>0,05$ & $p>0,05$ \\
Pain (VAS) & $r=0,78 ; p<0,05$ & $p=0,07 ; r=0,45$ \\
Pain (WOMAC) & $r=0,89 ; p<0,05$ & $p>0,05$ \\
Total WOMAC & $p>0,05$ & $p>0,05$
\end{tabular}

Conclusion: Our study showed that patients with obesity and knee OA have higher rates of mTOR expression, compared to patients with normal body weight. High mTOR expression correlates with the severity of knee pain in obese patients. Thus, the evaluation of mTOR expression in obese patients and knee OA plays an important role in predicting the severity of clinical manifestations of $O A$, and may influence the choice of personalized therapy tactics for such patients.

Disclosure of Interests: None declared

DOI: 10.1136/annrheumdis-2021-eular.3287 